\title{
Operationalizing the Development of the National Unified Health Research Agenda 2017-2022
}

\author{
Miguel Manuel C. Dorotan, Rafael Deo F. Estanislao, Reneepearl Kim P. Sales, \\ Maria Angeli C. Magdaraog and Lester Sam A. Geroy \\ Alliance for Improving Health Outcomes
}

\begin{abstract}
Introduction. The National Unified Health Research Agenda is a major document whose creation by the Philippine National Health Research Systems is mandated by Philippine Law. Multiple frameworks, methods, and approaches for the development of a health research agenda exists. Available literature on research priority setting have mostly focused on its methods, results, or implementation. There are limited studies on the operations management of conducting a national health research priority-setting exercise.
\end{abstract}

Objectives. This paper aims to describe the operational components utilized to develop the NUHRA 2017-2022 and provide lessons learned for future research priority setting endeavors.

Methods. This paper identified and categorized the operational components to develop the NUHRA 2017-2022 using the Enabler Criterion of the European Foundation for Quality Management's (EFQM) Excellence Model.

Results. Operationalizing the development of a national health research agenda requires the harmonization of multiple components. Having region-based staff involved in the production of a regional situational analysis improved its context and accuracy. The workload for each cluster team must be considered and anticipated by the management. Stakeholder engagement requires political, diplomatic, advocacy, marketing, management, and technical skills. The process requires building relationships with various institutions. In a largescale project, management processes must be in place to effectively track and monitor its progress.

Conclusion. Operations and management are usually a neglected area in research and development projects. In a bottoms-up research priority-setting approach, iteration and flexibility is needed which requires a lot of management skills and insight. Duration of the project, planning, resources available, and quality of outputs have a strong interconnected relationship.

Key Words: organizational management, health priorities, organizational innovation

\section{INTRODUCTION}

Corresponding author: Reneepearl Kim P. Sales, RMT, MLS(ASCPi)CM, MSC

Alliance for Improving Health Outcomes

62 West Avenue, Quezon City 1104, Philippines

Email: kimsales@aiho.org.ph
The National Unified Health Research Agenda (NUHRA) is a major document whose creation by the Philippine National Health Research Systems (PNHRS) is mandated by Philippine Law. The creation of a health research agenda maximizes the impact of research investments, strengthens national health research systems, and harmonizes research priorities even at the global level. ${ }^{1}$ Multiple frameworks, methods, and approaches for the development of a health research agenda exists. While there is no consensus on what constitutes best practice in a research priority setting exercise, an essential checklist developed in 2010 suggests that these exercises must be contextual, comprehensive, inclusive, informed, and include plans for implementation. ${ }^{1}$ The development framework of 
the NUHRA 2017-2022 adopted the Kingdon Multiple Streams Model. ${ }^{2}$ The framework aims to create a "policy window" that facilitates acceptability and adoptability of a policy being developed through identifying gaps and its solutions, assessing feasibility, analyzing local context, and determining public opinion or mood. An inclusive, realistic, and collaborative approach was also utilized in the development of the NUHRA 2017-2022, guided by the PNHRS guideline for producing health research priorities. ${ }^{3}$

Available literature on research priority setting have mostly focused on its methods, results, or implementation. There are limited studies on the operations management of conducting a national health research priority-setting exercise. Recognizing the need to ensure the production of an informed, inclusive, realistic, and collaborative research agenda, this paper aims to describe the operational components utilized to develop the NUHRA 2017-2022. The paper also provides lessons learned for future research priority setting endeavors.

\section{Development of NUHRA 2017-2022}

The project was conducted over a period of nine months, from February to November 2017. Figure 1 summarizes the activities conducted over the duration of the project. February to April 2017 was dedicated to the development of technical papers and regional situationers. These papers provided global, national, and regional context that included current health objectives, policy trends, health challenges, and status of the health research system. Preparation of materials and kits for the regional consultations was also done during this period. The kit included presentation slides, infographics, and other regional facilitation training tools. In addition, stakeholders for regional consultations were mapped and invited. Seventeen regional consultations and one national consultation were held from March to June 2017, with a total of 378 stakeholders who participated. From June to November 2017, project activities were dedicated to NUHRA production, which included writing and branding of the document.

\section{METHODS}

This paper identified and categorized the operational components to develop the NUHRA 2017-2022 using the Enabler Criterion of the European Foundation for Quality Management's (EFQM) Excellence Model (Table 1). This model asserts that there are five enablers required for an organization to implement a project: 1) strategy, 2) people, 3) leadership, 4) partnerships and resources, and 5) processes, products, and services. ${ }^{4}$
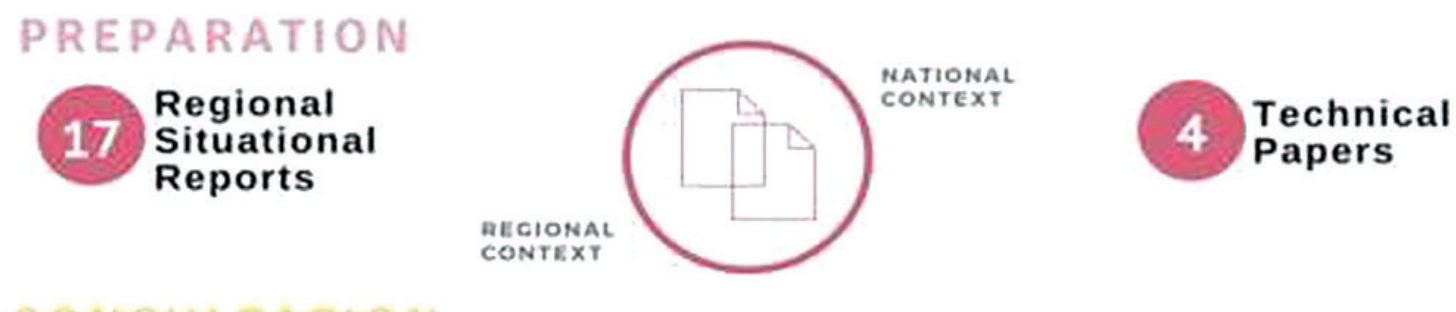

\section{CONSULTATION}

Regional Consultations

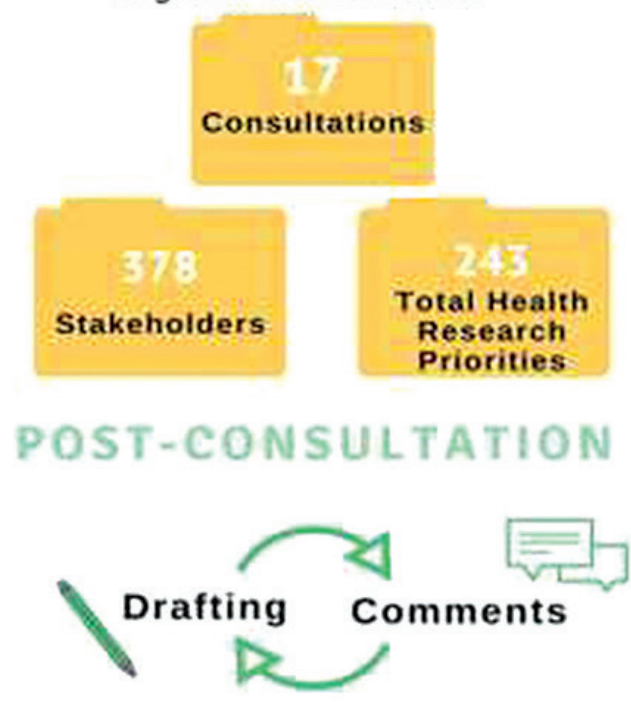

National Consultations
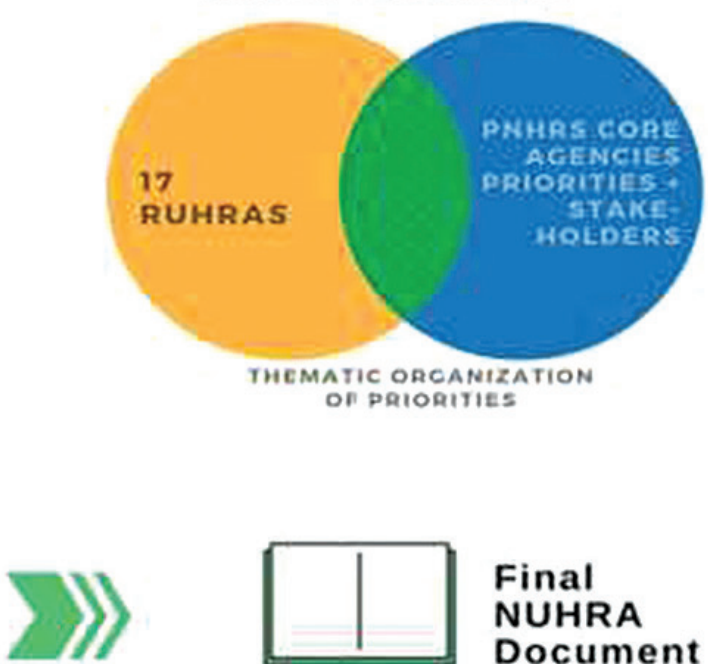

Final

NUHRA

Document

Figure 1. Summary of activities for the development of NUHRA 2017-2022. 
Table 1. Enabler criterion of the EFQM Excellence Model and their definitions

\begin{tabular}{ll}
\hline Strategy & $\begin{array}{l}\text { Policies, plans, objectives and processes are } \\
\text { developed and deployed to deliver the strategy. }\end{array}$ \\
\hline People & $\begin{array}{l}\text { Excellent organizations value their people } \\
\text { and create a culture that allows the mutually } \\
\text { beneficial achievement of organizational and } \\
\text { personal goals. They develop the capabilities } \\
\text { of their people and promote fairness and } \\
\text { equality. They care for, communicate, reward } \\
\text { and recognize, in a way that motivates people, } \\
\text { builds commitment and enables them to use } \\
\text { their skills and knowledge for the benefit of } \\
\text { the organization. }\end{array}$ \\
& $\begin{array}{l}\text { Leaders are flexible and enable the organization } \\
\text { to anticipate and react in a timely manner to } \\
\text { ensure the on-going success of the organization. }\end{array}$ \\
\hline Leadership & $\begin{array}{l}\text { The planning and management of external } \\
\text { partnerships, suppliers, and internal resources } \\
\text { in order to support strategy and policies and the } \\
\text { effective operation of processes. }\end{array}$ \\
\hline $\begin{array}{l}\text { Partnerships } \\
\text { and resources }\end{array}$ & $\begin{array}{l}\text { The design, management, and improvement } \\
\text { of processes to generate increasing value for } \\
\text { customers and other stakeholders }\end{array}$ \\
\hline Processes, &
\end{tabular}

\section{Operational Components}

\section{Strategy and People}

The development framework of the NUHRA 20172022 envisioned an inclusive, realistic, and collaborative bottoms-up approach in which the development of Regional Unified Health Research Agendas (RUHRA) served as inputs to the creation of a NUHRA. This entailed the conduct of 17 regional consultations involving multisectoral stakeholders. To operationalize this, a cluster system was implemented that divided the country into four main areas
(Figure 2): Northern Luzon, Southern Luzon, Visayas, and Mindanao. The Northern Luzon cluster covered Regions 1, 2, 3, and CAR; Southern Luzon covered regions 4A, 4B, 5, and NCR; Visayas covered regions 6, 7, and 8; and Mindanao covered regions 9, 10, 11, 12, CARAGA, and ARMM. Each cluster was assigned to a cluster team composed of a cluster lead, research assistant, and a regionbased coordinator. The teams were tasked to map possible stakeholders to invite to the regional consultation, produce a regional situationer, facilitate the regional consultation and write the first draft of the RUHRA. The clusters coordinated with the Regional Health Research and Development Consortia (RHRDC) and the regional government offices to complete these tasks.

\section{Leadership}

A core team of five people was responsible for the overall project management and quality assurance of outputs. This was composed of the Project Lead, Project Manager, Assistant Project Manager, Technical Lead, and Assistant Technical Lead.

The Technical Lead and the Assistant Technical Lead formed the Technical Team and oversaw the development of the technical papers, consultation design, data analysis, and synthesis of RUHRAs to develop the NUHRA. The Technical Team had a link to each cluster team, providing support on the framework, consultation methods, and feedback on the regional situationers and RUHRAs. The Project Manager and the Assistant Project Manager led the Operations Team and, with administrative support, directly monitored and guided the activities of the cluster teams. In addition, they were responsible for the financial and human resources management of the project.

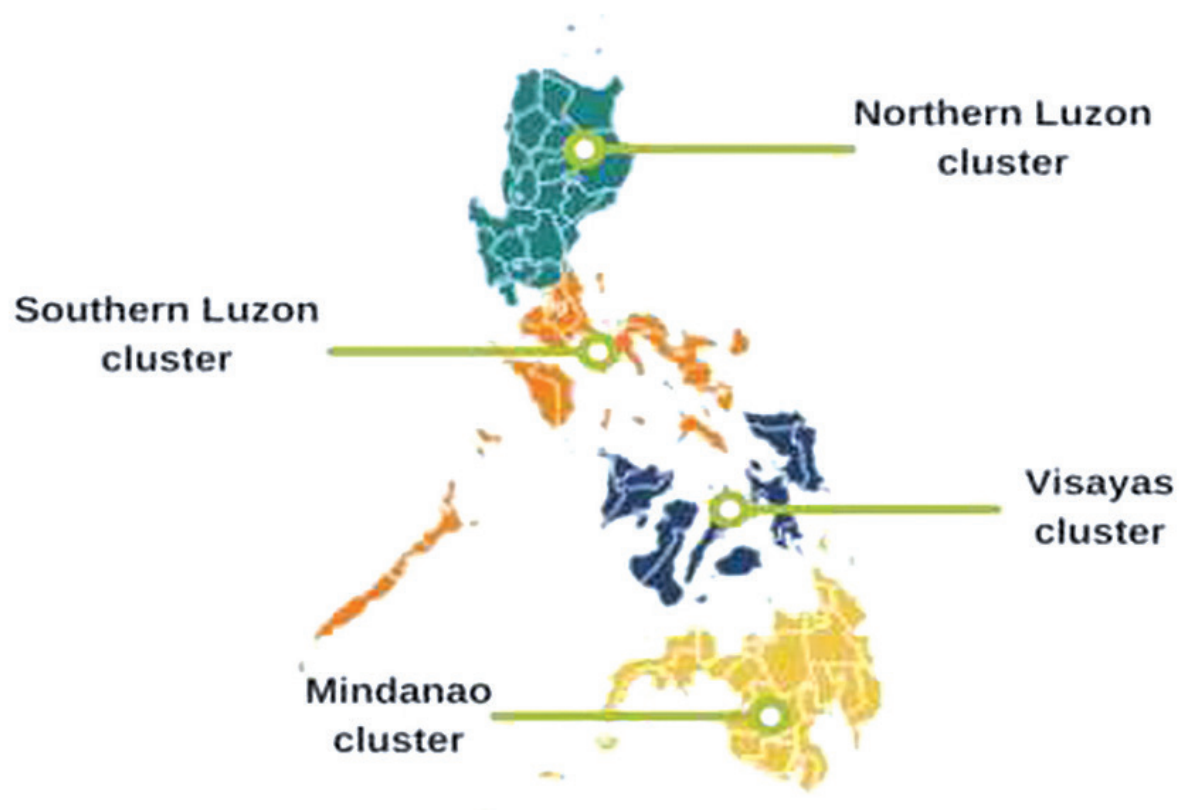

Figure 2. Cluster system map. 
A weekly to bi-weekly meeting of the core team provided updates on deliverables and concerns regarding project implementation. Both technical outputs and operational concerns were discussed and possible solutions assessed.

\section{Partnerships and Resources}

Internal resource management tasks included daily budget projection, weekly and monthly reviews, budget readjustments, accounting, liquidation, and other related functions. A detailed budget plan, attached to the workplan and staffing, was developed as soon as the project was approved. A budget realignment was requested on the fifth month of the project after highly-funded activities were completed.

The development of a NUHRA necessitated the engagement of multisectoral stakeholders. The project conducted external meetings to coordinate with these stakeholders, with different logistical requirements. Regional coordination meetings and courtesy visits were conducted by the cluster teams in the regional office of government agencies, research consortium, universities, and other stakeholders. This meeting introduced the project to the stakeholders, garnered their support for the regional consultation, and provided input to the regional situationer to be presented in the regional consultations.

The Research Agenda Committee (RAC) is the national decision-making body responsible for the NUHRA. It is composed of representatives from the Department of Science and Technology, Department of Health, Commission on Higher Education, and the University of the Philippines National Institutes of Health. Members of RAC assigned to oversee the development of NUHRA 2017-2022 were provided with weekly updates from the core team, supplemented with quarterly meetings with the committee. The final draft of the NUHRA was presented to the RAC for their official approval and adoption.

\section{Processes, products, and services}

Two main approaches were implemented by the core team to ensure that the envisioned approach would be realized and to effectively manage, track, and monitor the project progress. First is the creation of a project dashboard with three main segments: workplan, operations summary, and staff tracking. A list of activities, subactivities, and the Person/s-in-Charge were laid out in the Workplan segment of the project dashboard. This provided an overview of all the tasks and activities of the project until its completion. This sheet is static and is revised only when there were major revisions to project activities. The Operations Summary provided an overview of the status of milestones set for all stages of the project. Deliverables of the core team and the cluster teams were listed, along with its completion status. This sheet is dynamic, and updates are immediately reflected on the dashboard. Staff Tracking listed all engaged staff for the duration of the project. This included the core team, technical team, operations team, and the cluster teams. It served as a directory and monitoring tool for all tasks and activities assigned to project staff. It also ensured that all staff were properly briefed and prepared. This sheet is seldom revised but is used whenever additional staff is needed to be onboarded.

The second main approach was the use of an online communication portal. All project staff were required to register and use the portal as the standard communication tool for the duration of the project. Through this platform, staff provided updates, submitted weekly reports and other deliverables, were given feedback, and coordinated with project staff located in different regions.

\section{CONCLUSIONS AND LESSONS LEARNED}

Operationalizing the development of a national health research agenda requires the harmonization of multiple components. Following the Kingdon Model along with envisioning an inclusive, realistic, and collaborative approach, the core team projected the necessary activities and strategized the best way to mobilize available resources to achieve the project objectives.

Having region-based staff involved in the production of a regional situational analysis improved its context and accuracy. The workload for each cluster team must be considered and anticipated by the management. During the development of the NUHRA 2017-2022, staffing adjustments were made to facilitate work in Mindanao. An additional research assistant was added, with one assigned to Eastern Mindanao (Regions 10, 11, Caraga) and another to Western Mindanao (Regions 9, 12, ARMM). This was due to the greater size of Mindanao compared to the other clusters, which had four or less regions each. In addition, the large scale of the project required the involvement of another institution to aid in logistics of travel and accommodation for consultation with participants.

The identification of the Core Team was crucial to the success of the project. Its senior members (Project Lead, Project Manager, and Technical Lead) had wide experience in public health, global health systems, national level planning, emergency preparedness and response, policy development, and research. The launch of the NUHRA 2017-2022 was slated eight months after the start of its development. Thus, urgency and timeliness were imperative to produce deliverables according to the project timeline. The core team's experience led them to implement operational and communication protocols derived from emergency response systems.

Stakeholder engagement requires political, diplomatic, advocacy, marketing, management, and technical skills. The process requires building relationships with various institutions. Persons and institutions responsible, accountable, consulted, and informed for the agenda should be well identified. The cluster system, with a region- 
based lead and coordinator, was a good strategy to engage stakeholders. In addition, it was important to maintain close coordination with the RAC as the national body in charge of the NUHRA. Engaging stakeholders ensured ownership and support for the subsequent implementation of the RUHRA and NUHRA.

In a largescale project, management processes must be in place to effectively track and monitor its progress. The utilization of an online project dashboard that contains the status of deliverables, activities, and tasks assigned to each project staff eased the monitoring of a project with activities simultaneously conducted all over the country. Monitoring and tracking were also eased by the use of a single portal by all project staff that served as the project's daily communication tool for updates, submissions, and feedback. It is important to note that the dashboard is useful only if the basis for its design is the work schedule and organizational structure of the team.

The management team needs to be clear on measurable indicators for acceptable performance. For example, an acceptable output is one that has passed review of all core team members. Only the information necessary for decision-making at the management level must be identified and collected.

Operations and management are usually neglected areas in research and development projects. In a bottoms-up research priority-setting approach, iteration and flexibility is needed which requires a lot of management skills and insight. Duration of the project, planning, resources available, and quality of outputs have a strong interconnected relationship. To nurture and sustain management talent, government agencies and funders should invest in project management and operations in the areas of budget and capacity-building.

\section{Acknowledgment}

The authors acknowledge the contributions of Professor Marilyn Ellorin-Crisostomo and Dr. Katherine Villegas-Reyes.

\section{Statement of Authorship}

All authors approved the final version submitted.

\section{Author Disclosure}

All authors declared no conflict of interest.

\section{Funding Source}

This paper was funded by the Philippine Council for Health Research and Development.

\section{REFERENCES}

1. Viergever RF, Olifson S, Ghaffar A, Terry RF. A checklist for health research priority setting: nine common themes of good practice. Health Res Policy Syst. 2010 Dec 15; 8(36). doi: https://doi.org/10.1186/14784505-8-36.

2. Kingdon J. Agendas, Alternatives, and Public Policies. Boston: Little Brown;1984.

3. Philippine National Health Research System. Guidelines for Health Research Prioritization. 2016.

4. European Foundation for Quality Management. An Overview of the EFQM Excellence Model Brussels, Belgium: EFQM; 2012 